\title{
Commentaries
}

\section{EVOLUTION AND ADDICTION}

\section{Proximate explanations}

An evolutionary approach to behavior is wonderful because is poses fundamentally new questions. It is also difficult, however, precisely because these questions are new and therefore hard to formulate clearly and even harder to answer. The papers in this special issue (Dudley 2002; Gerald \& Higley 2002; Hill \& Chow 2002; Lende \& Smith 2002; Newlin 2002; Panksepp et al. 2002; Sullivan \& Hagen 2002) tackle the problem of substance abuse from an evolutionary perspective. They range from deductions about risk-taking based on life history theory to adaptationist hypotheses about the possibility that drug use has been useful in our past, and therefore selected for. Those who are unfamiliar with such questions will appreciate a sketch of the larger framework in which evolutionary and proximate explanations are both needed to fully understand any biological trait.

Most substance abuse research has addressed the problem of why some people abuse drugs and others do not. The core challenge has been to explain individual differences. This focus arises from practical concerns-if we know the factors that predict who will abuse substances we should be able to change them and thus assist prevention and treatment. Much of the debate in the field has been between those who emphasize the importance of genetic differences and those who call attention to the roles of life experiences and psychological factors. Much of the progress has come from studies of the brain mechanisms - how they are changed by drugs and how they lead to drug craving and using. All of these questions and answers are fundamentally the same, however, in that they examine how things work. Together, they constitute what evolutionary biologists call a proximate explanation for substance abuse.

\section{Why are we all vulnerable?}

What they leave out, however, is the separate question of why all humans are vulnerable to substance abuse. This is a different kind of question, an evolutionary question about why we are all the same in this regard.
Its answer is to be found in the forces of natural selection that shaped the brain (Nesse 1994). Substance abuse happens because natural selection shaped behavior regulation mechanisms that are based on chemical transmitters. Not only is it unsurprising that certain substances stimulate the system in ways that generate escalating drug taking, it seems nearly inevitable. While extremely simple, this principle is also important because it changes our perspective. Drug taking is what we should expect. The amazing thing to be explained is that substance abuse is so limited. Instead of asking what is wrong with people who are especially vulnerable to drug use, we should ask why some people do not use drugs, and especially why some people can use drugs and then stop.

This perspective also helps to explain why substance abuse is so devastating. If it took simply some time and money it would be more like gambling, video games or other unproductive diversions. Psychoactive substances, however, disrupt the very emotions that evolved to regulate our behavior (Nesse et al. 1997). They arouse reward mechanisms artificially, thus stimulating the circuits that are normally fired by an event that provides a huge gain in fitness; but they provide no fitness gain, they simply create an illusion. People who have few sources of pleasure in their daily lives are understandably more vulnerable to substance abuse. Treatments directed to the person's whole life and place in a social network are essential because restoring normal sources of satisfaction may reduce the pull of drugs. Interestingly, an evolutionary approach supports a comprehensive and integrated search for the proximate causes of substance abuse, and it supports psychosocial treatments in conjunction with pharmacological interventions. Widespread adoption of an evolutionary view of addiction could be enormously useful by simply defusing useless controversies between different camps in the field, and by providing a biological foundation for integrated treatment models.

\section{Why do plants carry drugs?}

The second major question that emerges from an evolutionary perspective on substance abuse is why drugs of abuse exist ready-made in plants. They are there for the 
good reason that plants cannot run away from other organisms that want to eat them, so they have to rely on chemical defenses. Over hundreds of millions of years, plant biochemistry has been shaped to make chemicals that protect from herbivores. Some such chemicals, such as those that clog the mouthparts of insects, do not stop us for a minute. Others, such as tannins, impair digestion for both insects and mammals; but some chemicals, such as caffeine, cocaine and opiates are present in plants specifically because they interact with animal nervous systems. A mouse that tries to eat a coffee bean does not just suffer insomnia; it is likely to become very sick indeed. A leaf-miner that tries to eat a tobacco leaf does not become high, it dies. Nicotine, opiates and cocaine are potent toxins. Plants with such toxins have a selective advantage.

There is, however, another way that plants can obtain an advantage, and that is by offering something attractive. The nectar of flowers lures bees who spread pollen, and the tasty flesh of fruits means that they are eaten so the seeds are spread far from the plant, often reaching the ground in conjunction with rich nutrients. Are there any plants that contain drugs because they attract caretakers? Certainly tobacco, cocaine and opium poppies would not be nearly so widespread were it not for human desires for their chemicals. It seems likely to me that these chemicals evolved as toxins and the plants that make them simply happen to have had recent good luck in that they lead to human cultivation, but others have different ideas about this.

A related question is whether exposure to drugs has been a force of natural selection for humans. A mutated version of the gene that codes for alcohol dehydrogenase $(\mathrm{ALDH} 2 * 2)$ has long been thought to offer a selective advantage by making its bearers sick when they drink alcohol. The relative frequency of this mutation in Asian populations, as high as $50 \%$, may be explained by the centuries during which distillation has been available there. The other side of this idea is advocated by those who suggest in this issue that drug taking has benefited humans for long enough to have shaped special aspects of our psychology to reap those benefits and minimize the costs.

Whether or not selection has shaped some genes that prevent or confer benefits from drug use, it seems clear that we were never designed to cope with ready access to a wide variety of pure drugs. In the ancestral environment drugs were harder to obtain and harder to store and use. Now, every decade brings us new drugs, new methods of transporting them and new routes of administration. In this sense, drug abuse is a disease of civilization. This provides yet another source of wonder that drug use is not far more common than it is. Most young people now assume that normal people can resist addic- tion and only other weak people succumb. If the public perception was based instead on an evolutionary perspective that we are all designed in ways that make us vulnerable, perhaps this could foster a more realistic view of the risks.

\section{Looking for an integrative framework}

The questions addressed in this special issue are new and challenging. The perspective they offer is not an alternative, but complementary to other knowledge about substance abuse. I am not at all sure they will lead quickly to new ways to prevent or treat substance abuse, but they certainly do offer a useful foundation for understanding what substance abuse is, and why we all should be interested in the evolutionary reasons why we are vulnerable. Even now, however, this understanding may well provide a framework that can integrate different perspectives and provide a foundation for comprehensive programs of treatment and sensible public policies.

RANDOLPH M. NESSE
The University of Michigan
Departments of Psychiatry and Psychology
and Institute for Social Research
426 Thompson Street
Ann Arbor
MI 48104, USA

\section{References}

Dudley, R. (2002) Fermenting fruit and the historical ecology of ethanol ingestion: is alcoholism in modern humans an evolutionary hangover? Addiction, 97, 381-388.

Gerald, M. S. \& Higley, J. D. (2002) Evolutionary underpinnings of excessive alcohol consumption. Addiction, 97, $415-425$.

Hill, E. M. \& Chow, K. (2002) Life-history theory and risky drinking. Addiction, 97, 401-413.

Lende, D. H. \& Smith, E. O. (2002) Evolution meets biopsychosociality: an analysis of addictive behavior. Addiction, 97, 447-458.

Nesse, R. M. (1994) An evolutionary perspective on substance abuse. Ethology and Sociobiology, 15, 339-348.

Nesse, R. M., Kent, C. \& Berridge (1997) Psychoactive drug use in evolutionary perspective. Science, 278, 63-66.

Newlin, D. B. (2002) The self-perceived survival ability and reproductive fitness (SPFit) theory of substance use disorders. Addiction, 97, 427-445.

Panksepp, J., Knutson, B. \& Burgdorf, J. (2002) The role of brain emotional systems in addictions: a neuro-evolutionary perspective and new 'self-report' animal model. Addiction, 97. 459-469.

Sullivan, R. J. \& Hagen, E. H. (2002) Psychotropic substanceseeking: evolutionary pathology or adaptation? Addiction, 97 , 389-400. 


\section{TAKING DARWIN SERIOUSLY: MORE THAN TELLING JUST SO STORIES}

If you accept, as I do, that the modern Neo-Darwinian evolutionary account is approximately correct, you believe that many human behavioural traits and characteristics were selected for during the period when our early hominid ancestors were hunter-gatherers on the African savannah. If the Darwinian account is not true, it is surely a remarkable coincidence that humans share more than $99 \%$ of their genome with chimpanzees who inhabit the same contemporary environment.

One consequence of an evolutionary theory of our origins is elegantly pointed out by Sullivan \& Hagen (2002): that humans, and our hominid ancestors, have had a long exposure to psychoactive compounds in their plant diet. Dudley (2002) suggests that our longest experience has been with ethanol in over-ripe and fermenting fruit. He also notes the similarity between the BACs achieved in the ancestral environment and the level of alcohol use that epidemiological evidence suggests may protect middle-aged males from premature death caused by cardiovascular disease. It seems unlikely, however, that low-level alcohol use would have conferred any adaptive advantage in the ancestral environment because cardiovascular disease in middle life (and living beyond contemporary middle life!) were rare.

The archaelogical and ethnographic record summarized by Sullivan \& Hagen (2002) reveals the ingenuity of humans in extracting psychoactive substances from plants to use as food or medicine. An evolutionary account of human origins helps to makes sense of what would be two otherwise puzzling coincidences: (1) that until very recently all of the major psychoactive substances that have been historically used and abused by humans have come from plants; and (2) that the effects of these substances on the human and mammalian central nervous system mimic those of important neurotransmitters.

The fact that some humans become addicted to plant-based psychoactive substances is more of a puzzle, as Gerald \& Higley (2002) point out. Why is addiction to these psychoactive substances so common among humans if (as is likely): (a) addiction is partially under genetic control and (b) those who become addicted are at increased risk of dying prematurely and thereby reduce their chances of contributing to the future of their species?

One explanation is that some types of addiction do not reduce reproductive success. Cigarette smoking, for example, does not exert its adverse effects on the user's survival until after he or she has reproduced. A second possibility (suggested by Panksep and colleagues (2002) and others) is that addiction is the result of using pure psychoactive substances that are delivered in highly efficient ways by injection or smoking, and subvert brainreward systems in ways that were not possible in the ancestral habitat. People who become addicted to these drugs die at a much higher rate than their peers, but addiction has been present in human populations for too few generations to have been selected against.

A third possibility (proposed by Hill \& Chow, 2002) is that the early use of psychoactive substances is a highrisk strategy that increases the chance of reproduction among young males who most often adopt it. A strong correlate of the strategy 'live fast, die young (and leave a beautiful corpse)' is precocious sexual activity and teenage pregnancy which (before the advent of liberal abortion laws) increased their chances of reproducing. This hypothesis provides one explanation (but hardly the only one) for the high rates of alcohol and other drug use in adolescence that decline in early adult life under the influence of marriage, mortgages and children.

A critical question for evolutionary psychology is: how do we distinguish plausible evolutionary hypotheses about addictive behaviour from what Gould \& Lewontin (1979) have described as 'just so' stories: stories that provide seemingly plausible but untestable and empty 'explanations' of behaviour?

A major problem is that humans no longer live in the ancestral environment Even the descendants of huntergatherers live in environments that have been transformed by industrial civilization. Instead, we have to look for consilience of evidence from: archaeology on human consumption of plants; from 19th- and early 20thcentury ethnography on the use of plant-based drugs by hunter-gatherers; oral histories from the dwindling number of people whose parents and grandparents once lived as hunter gatherers; observational studies of other hominids in their natural environment; and experimental studies of the effects of drugs in other animals. The ultimate test will be whether evolutionary hypotheses improve our modest ability to assist problem drug users to desist from or reduce the harm caused by their drug use.

WAYNE HALL Professor and Director Office of Public Policy and Ethics Institute for Molecular Bioscience, University of Queensland St Lucia QLD 4072 Australia

\section{References}

Dudley, R. (2002) Fermenting fruit and the historical ecology of ethanol ingestion: is alcoholism in modern humans an evolutionary hangover? Addiction, 97, 381-388. 
Gerald, M. S. \& Higley, J. D. (2002) Evolutionary underpinnings of excessive alcohol consumption. Addiction, 97, 415-425.

Gould, S. J. \& Lewontin, R. (1979) The spandrels of San Marco. Proceedings of the Royal Society B, 205, 581-598.

Hill, E. M. \& Chow, K. (2002) Life-history theory and risky drinking. Addiction, 97, 401-413.

Panksepp, J., Knutson, B. \& Burgdorf, J. (2002) The role of brain emotional systems in addictions: a neuro-evolutionary perspective and new 'self-report' animal model. Addiction, 97, 459-469.

Sullivan, R. J. \& Hagen, E. H. (2002) Psychotropic substanceseeking: evolutionary pathology or adaptation? Addiction, 97 . 389-400.

\section{EVOLUTIONARY PSYCHOBIOLOGY: ANY RELEVANCE FOR THERAPY?}

There need be no doubt that substances with psychoactive effects are ancient and ubiquitous in this universe. The human form of the first enzyme in the breakdown pathway of ethanol, alcohol dehydrogenase, has been traced back 450 million years to the earliest bony fish. Radio astronomers are reported to have discovered a distant constellation whose atmosphere consists of billions of litres of ethanol vapour.

\section{Blame versus understanding}

Are there any implications for clinicians? Or does evolutionary biology provide us only with ways, albeit helpful, to apportion less blame to those who harm themselves and others by their use of substances? For example, we understand such people a little more, in that our species has benefited because certain impulsivity genes have lasted till this stage of our evolution: "while impulsivity may ultimately lead individuals to their untimely death in some environments, impulsivity may offer individuals selective advantages such as food access and mating opportunities to reproduce in other conditions [i.e. more uncertain or aversive]' (Gerald \& Higley 2002).

\section{Cognitions}

Because individual responsibility is such a building block of human society, the readers may reject these papers because of their apparent biological determinism. One rejoinder might be: 'But highly reflective organisms that always think before acting would probably not have a high probability of representing their genes in future generations' (Le Doux, quoted by Panksepp et al. 2002); and yet the explanations offered in these papers leave plenty of room for cognitions and intention, as well as genes and environment. For example, Newlin (2002) would not say, as a sociobiologist might, that Homo sapiens (male) drinks to display dominance over another member of the pack.
Newlin's SPFit theory proposes that the drinking causes a temporary enhancement of self-perceived power and reproductive potential. This phenomenon is probably highly accessible to the cognitive therapist, who can help the drinker evaluate and, if appropriate, challenge expectations and cognitions linked to drinking. (If they wish, the patient and therapist can then speculate whether those cognitions are best understood as learnt, or as generated by the effects of alcohol on the archaic 'fitness/ survival substrate'. The cortico-mesolimbic dopamine system was previously given the interim appellation 'reward circuits'. We now have a less tautological term.)

"Abused drugs "hijack" the motivational system that evolved to regulate survival and reproductive functions. This process occurs because drugs activate this system as if they promoted survival and reproduction when they actually do not' (Newlin 2002). Once again, this may guide the cognitive therapist towards cognitions that could be checked and challenged: 'You feel more sexy when drinking. Lets review the evidence about your sexual success under the influence of alcohol.

\section{The early environment}

Two of the essential phenomena in mammalian survival, attachment, and place in the social rank, have had a special role in evolutionary psychiatry. Indeed, Stevens \& Price (1996) offer 'disorders of attachment and rank' as one category of psychiatric disorders, which includes depression, personality disorder and anxiety and phobic disorders. Hill \& Chow (2002) have defined empirically another factor in the development of psychiatric disorder: family unpredictability. Research inspired by this perspective has assessed family unpredictability as a dimension of the developmental environment. Family unpredictability leads to risk-taking behaviour, acting in addition to, and as a factor distinct from, amount of environmental resources.

This dimension is particularly relevant to characterizing the functioning of families where a parent is alcohol dependent, and therefore children encounter little consistency from day to day. Loss of routines and consistent parental family roles during a critical period in development has long-term implications for behavior, emotional responses, and social cognition. Early environmental characteristics might lead to a cognitive set or mental model of the future as uncertain, leading to rejection of future rewards and benefits as unreliable ... Altering environmental predictability may be a feasible target for intervention in family therapy that could produce long lasting results. 
This has already been endorsed: almost the only primary preventive strategy for youth alcohol misuse which meets Cochrane criteria for efficacy (Foxcroft et al. 2002 ) is just such a family programme (Spoth et al. 1999).

Risky behaviour, common in the offspring of such families, can also be viewed with less opprobrium, if we realize that evolutionary theory predicts that where present environments, and therefore possibly future environments, are unstable, early risk-taking, including early reproduction, is in evolutionary terms the effective choice of behaviour.

\section{Simplistic extrapolations}

Extrapolations of biological findings to therapy will have to be tested carefully. Too-ready application of animal behaviour findings to the clinic may be dangerous. Clinicians may have leapt too readily to give specific serotonin uptake inhibitors (SSRIs) to alcohol-dependent patients, a practice which especially in primary care is now common in many countries, and this has its origins in studies of drinking and stress in laboratory animals. It seemed to be supported by the later work (reviewed by Gerald \& Higley 2002) in monkeys in near-natural circumstances. One example of this research was that monkeys with low rates of affiliative interactions tended to have low CSF 5-HIAA concentrations. Infrequent social interactions and low CSF 5-HIAA concentrations were predictive of high alcohol consumption, and when the subjects that were prone to consume high volumes of alcohol interacted socially, their interactions were brief and appeared furtive.

This and other work seems to relate easily to humans. Low CSF 5HIAA in alcoholic patients, especially Type 2 patients, had been found and the shorthand interpretation 'a CNS serotonin deficiency' was attributed to some alcoholics. It was, however, too simplistic to think that Type 2 patients' drinking would therefore become less risky if the patient was prescribed an SSRI. In fact, it seems that Type 2 patients may have a less favourable outcome with SSRIs than placebo (Kranzler et al. 1996; Chick et al. 2002), or show no response while Type 1 patients benefit (Pettinati et al. 2000). And, of course, while higher CSF 5HIAA in these Gerard's studies correlated with increased reproductive fitness, SSRIs in humans are notable for their inhibition of sexual performance. However, this is a criticism of our under- standing and use of human psychopharmacotherapy, not of animal behavioural ecology.

\section{Not a dodo}

These exhilarating papers show how fitness can result from mixing genes across academic specialities. Addiction science that is isolated will die out.

\section{JONATHAN CHICK Lothian Primary Care NHS Trust Alcohol Problems Clinic 35 Morningside Place Edinburgh EH1O 5HF}

$U K$

\section{References}

Chick, J., Aschauer, H. \& Hornik, K. (2002) Efficacy of fluvoxamine in preventing relapse in alcohol dependence: a oneyear, double blind, placebo-controlled multicentre study with analysis by typology (in preparation).

Foxcroft, D. R., Lister-Sharp, D., Lowe, G., Breen, R. \& Ireland, D. (2002) Cochrane Review Issue (in preparation).

Gerald, M. S. \& Higley, J. D. (2002) Evolutionary underpinnings of excessive alcohol consumption. Addiction, 97, 415-425.

Hill, E. M. \& Chow, K. (2002) Life history theory and risky drinking. Addiction, 97, 401-413.

Kranzler, H. R., Burleson, J. A., Brown, J. \& Babor, T. F. (1996) Fluoxetine treatment seems to reduce the beneficial effect of cognitive-behavioural therapy in Type B alcoholics. Alcoholism: Clinical and Experimental Research, 20, 1534 1541.

Newlin, D. B. (2002) The self-perceived survival ability and reproductive fitness (SPFit) theory of substance use disorders. Addiction, 97, 427-445.

Panksepp, J., Knutson, B. \& Burgdorf, J. (2002) The role of brain emotional systems in addictions: a neuro-evolutionary perspective and new 'self-report' animal model. Addiction, 97, 459-469.

Pettinati, H. M., Volpicelli, J. R., Kranzler, H. R., Luck, G., Rukstalis, M. R. \& Cnaan, A. (2000) Sertraline treatment for alcohol dependence: interactive effects of medication and alcoholic subtype. Alcoholism: Clinical and Experimental Research, 24, 1041-1049.

Spoth, R., Lopez-Reyes, M., Redmond, C. \& Shin, C. (1999) Assessing a public health approach to delay onset and progression of adolescent substance use: latent transition and log-linear analyses of longitudinal family preventive intervention outcomes. Journal of Consulting and Clinical Psychology, 67, 619-630.

Stevens, A. \& Price, J. (1996) Evolutionary Psychiatry: A New Beginning. London: Routledge. 